\title{
Generalization of the Newman-Shapiro isometry theorem and Toeplitz operators. II
}

\author{
by \\ DARIUSz CichoŃ (Kraków)
}

\begin{abstract}
The Newman-Shapiro Isometry Theorem is proved in the case of SegalBargmann spaces of entire vector-valued functions (i.e. summable with respect to the Gaussian measure on $\mathbb{C}^{n}$ ). The theorem is applied to find the adjoint of an unbounded Toeplitz operator $T_{\varphi}$ with $\varphi$ being an operator-valued exponential polynomial.
\end{abstract}

1. Introduction. The study of Segal-Bargmann spaces (also called Fock spaces) dates back to early 60's, when the papers by Segal [23] and Bargmann [1] were published. They introduced a Hilbert space of entire functions summable with respect to the Gaussian measure as a natural model for Canonical Commutation Relations. This approach corresponds to Fock's results [13] in Quantum Mechanics. It seems that Newman and Shapiro [21, 22] discovered those spaces independently, motivated by Fischer's work on differential operators. It turned out that the Segal-Bargmann space provides a model for differential and pseudodifferential operators, which in these settings are strictly related to Toeplitz operators. We give a list of references, which could be twice as long, showing that the subject of Segal-Bargmann spaces and Toeplitz operators defined in them was intensively studied during the last century.

The isometry theorem, which is the main subject of the present paper, was first given in [22]. It turned out to be a powerful tool in the study of analytic Toeplitz operators. Our efforts focused on generalizing the theorem to vector-valued functions. Part of the job was done in [9] and what we present here may be regarded as a sequel. Another recent paper devoted to this subject [10] provides an exposition of the spectral synthesis problem in connection with Teoplitz operators. It is worth pointing out that the isometry theorem was also established for abstract operators (cf. [17, 18]), but it seems that this general idea would not be beneficial in our approach.

2000 Mathematics Subject Classification: Primary 47B35; Secondary 46E20, 46E40.

This research is supported by KBN under grant no. 2 PO3A 00417. 
We organized the material to make it as self-contained as possible, though we have to repeat some material from [9] without proofs.

2. Prerequisites. In what follows, $\mathcal{H}$ and $\mathcal{K}$ stand for separable Hilbert spaces. Basic properties of functions taking values in $\mathcal{H}$ or $B(\mathcal{H}, \mathcal{K})$ such as analyticity, measurability or integrability are understood in a weak sense (cf. [7]). Consider $L^{2}(\mu) \otimes \mathcal{H}$, the Hilbert space of all complex Borel functions taking values in $\mathcal{H}$ which are square-integrable on $\mathbb{C}^{n}$ with respect to the measure $\mu$ given by the formula $d \mu(z)=\pi^{-n} e^{-\|z\|^{2}} d V(z)$, where $V$ is the Lebesgue measure in $\mathbb{C}^{n}$ and $\|z\|^{2}=\left|z_{1}\right|^{2}+\ldots+\left|z_{n}\right|^{2}$ for $z=\left(z_{1}, \ldots, z_{n}\right) \in$ $\mathbb{C}^{n}$. The inner product in $L^{2}(\mu) \otimes \mathcal{H}$ is given by

$$
\langle f, g\rangle_{(\mathcal{H})}=\int_{\mathbb{C}^{n}}\langle f(\zeta), g(\zeta)\rangle d \mu(\zeta), \quad f, g \in L^{2}(\mu) \otimes \mathcal{H},
$$

where $\langle\cdot, \cdot\rangle$ stands for the inner product in $\mathcal{H}$. The norm induced by this inner product is denoted by $\|\cdot\|_{(\mathcal{H})}$. The Segal-Bargmann space $\mathcal{B}_{n} \otimes \mathcal{H}$ (for short $\mathcal{B} \otimes \mathcal{H})$ is a closed subspace of $L^{2}(\mu) \otimes \mathcal{H}$ consisting of all entire functions belonging to $L^{2}(\mu) \otimes \mathcal{H}$. We denote $P \otimes I_{\mathcal{H}}$ the orthogonal projection of $L^{2}(\mu) \otimes \mathcal{H}$ onto $\mathcal{B} \otimes \mathcal{H}$. We will use the following identifications: $L^{2}(\mu)=$ $L^{2}(\mu) \otimes \mathbb{C}, \mathcal{B}=\mathcal{B} \otimes \mathbb{C},\langle\cdot, \cdot\rangle=\langle\cdot, \cdot\rangle_{(\mathbb{C})},\|\cdot\|=\|\cdot\|_{(\mathbb{C})}$ and $P=P \otimes I_{\mathbb{C}}$.

Given $f: \mathbb{C}^{n} \rightarrow \mathbb{C}$ and $h \in \mathcal{H}$ we define $(f \otimes h)(z):=f(z) h, z \in$ $\mathbb{C}^{n}$. Put $e_{a}(z):=e^{z \cdot \bar{a}}$, where $z \cdot a:=\sum_{k=1}^{n} z_{k} a_{k}$ and $\bar{a}:=\left(\bar{a}_{1}, \ldots, \bar{a}_{n}\right)$ for $a=\left(a_{1}, \ldots, a_{n}\right) \in \mathbb{C}^{n}$ and $z=\left(z_{1}, \ldots, z_{n}\right) \in \mathbb{C}^{n}$. It can be checked that $\langle f(z), h\rangle=\left\langle f, e_{z} \otimes h\right\rangle_{(\mathcal{H})}$ for $f \in \mathcal{B} \otimes \mathcal{H}, h \in \mathcal{H}$ and $z \in \mathbb{C}^{n}$ (cf. [9]), which is referred to as the reproducing property for $\mathcal{B} \otimes \mathcal{H}$. By the Schwarz inequality we deduce that $\|f(z)\| \leq\|f\|_{(\mathcal{H})} e^{\|z\|^{2} / 2}, z \in \mathbb{C}^{n}$, for all $f \in \mathcal{B} \otimes \mathcal{H}$.

We denote by $\mathcal{P}\left(=\mathcal{P}_{n}\right)$ the space of all analytic polynomials in $\mathbb{C}^{n}$, which is a dense subset of $\mathcal{B}$. The sequence $f_{k}(z):=z^{k} / \sqrt{k !}, k \in \mathbb{N}^{n}, z \in \mathbb{C}^{n}$, forms an orthonormal basis for $\mathcal{B}$, where according to the standard multiindex notation $z^{k}:=z_{1}^{k_{1}} \ldots z_{n}^{k_{n}}, k !:=k_{1} ! \ldots k_{n}$ ! and $|k|:=k_{1}+\ldots+k_{n}$ for all $z=\left(z_{1}, \ldots, z_{n}\right) \in \mathbb{C}^{n}$ and $k=\left(k_{1}, \ldots, k_{n}\right) \in \mathbb{N}^{n}(\mathbb{N}=\{0,1,2, \ldots\})$.

Write $\mathcal{P} \otimes \mathcal{H}$ for the space of polynomials (in $\mathbb{C}^{n}$ ) taking values in $\mathcal{H}$, i.e. functions of type $\sum_{j=0}^{K} p_{j} \otimes h_{j}$, where $p_{j} \in \mathcal{P}_{n}, h_{j} \in \mathcal{H}$ and $K \in \mathbb{N}$. The space of operator-valued polynomials, denoted by $\mathcal{P} \otimes B(\mathcal{H}, \mathcal{K})$, can be defined analogously. Given $p \in \mathcal{P} \otimes B(\mathcal{H}, \mathcal{K}), p(z)=\sum_{|j|<K} A_{j} z^{j}, A_{j} \in B(\mathcal{H}, \mathcal{K})$, $z \in \mathbb{C}^{n}$, define $p^{\#} \in \mathcal{P} \otimes B(\mathcal{K}, \mathcal{H})$ via $p^{\#}(z)=p(\bar{z})^{*}, z \in \mathbb{C}^{n}$, and a differential operator $(p(D) F)(z)=\sum_{|j|<K} A_{j} D^{j} F(z), z \in \mathbb{C}^{n}$, where $F: \mathbb{C}^{n} \rightarrow \mathcal{H}$ is an analytic function (here $D^{j}=\partial^{|j|} / \partial z_{1}^{j_{1}} \ldots \partial z_{n}^{j_{n}}$ for $j=\left(j_{1}, \ldots, j_{n}\right)$ ).

We adhere to the following convention concerning the "dot" notation: $\|\varphi(\cdot)\|_{(\mathcal{H})}$ means the norm of the $\mathcal{H}$-valued function $z \mapsto \varphi(z)$, while $\|\varphi(\cdot)\|$ $\in Y$, where $Y$ is a set of functions, means that the function $z \mapsto\|\varphi(z)\|$ belongs to $Y$. 
Lemma 1. Let $f: \mathbb{C}^{n} \rightarrow \mathbb{C}$ be an entire function such that $f e_{z} \in L^{1}(\mu)$ for all $z \in \mathbb{C}^{n}$ and let $p \in \mathcal{P}$. Then $f(z)=\int_{\mathbb{C}^{n}} f(\zeta) e^{z \cdot \bar{\zeta}} d \mu(\zeta)$ for $z \in \mathbb{C}^{n}$ and

$$
\left(p^{\#}(D) f\right)(z)=\int_{\mathbb{C}^{n}} \overline{p(\zeta)} f(\zeta) e^{z \cdot \bar{\zeta}} d \mu(\zeta), \quad z \in \mathbb{C}^{n} .
$$

In particular, this holds for $f$ of the form $q(D) g$, where $g \in \mathcal{B}$ and $q \in \mathcal{P}$.

Proof. Cf. [9, Lemma 2.3].

Given $f \in L^{2}(\mu)$ we define

$$
G_{f}(z, w)=e^{-z \cdot w} \int_{\mathbb{C}^{n}} f(\zeta) e^{z \cdot \bar{\zeta}+w \cdot \zeta} d \mu(\zeta), \quad z, w \in \mathbb{C}^{n} .
$$

Note that this integral is meaningful, because the integrand is a product of two $L^{2}(\mu)$-functions. Let $X_{a}$ denote the space of scalar functions $\varphi$ for which $|\varphi(z)| \leq C e^{\|z\|^{2} / 2-a\|z\|}, z \in \mathbb{C}^{n}$, with some $C \geq 0$. It was shown in [22] that $G_{f} \in \mathcal{B}_{2 n}$ and $\left\|G_{f}\right\|=\|f\|$ for $f$ of the form $\bar{\varphi} g$, where $\varphi \in \bigcap_{a>0} X_{a} \cap \mathcal{B}$ and $g \in \mathcal{B}$ are chosen so that $\varphi g \in \mathcal{B}$. The proof of the following theorem is based on ideas from [20], which were inspired by those in [22].

Lemma 2. Let $f: \mathbb{C}^{n} \rightarrow \mathbb{C}$ be a Borel function such that

$$
f(\cdot) e_{a}(\cdot) \in L^{1}(\mu) \quad \text { and } \quad f(\cdot) e_{a}(\cdot) e^{-\|\cdot\|^{2} / 2} \in L^{2}(\mu)
$$

for all $a \in \mathbb{C}^{n}$. Let $G_{f}(z, w)$ be defined by (1). Then $G_{f}$ is analytic and

$$
\int_{\mathbb{C}^{n}} \int_{\mathbb{C}^{n}}\left|G_{f}(z, w)\right|^{2} d \mu(z) d \mu(w)=\int_{\mathbb{C}^{n}}|f(z)|^{2} d \mu(z)
$$

with both integrals possibly infinite.

Proof. First observe that the integral in (1) is absolutely convergent because, for any $z, w \in \mathbb{C}^{n},\left|e^{z \cdot \bar{\zeta}+w \cdot \zeta}\right|=\left|e_{z+\bar{w}}(\zeta)\right|$ for $\zeta \in \mathbb{C}^{n}$, and $f e_{z+\bar{w}} \in$ $L^{1}(\mu)$. We now turn to analyticity of $G_{f}$. By Lemma 1.1 of [19], $G_{f}(\cdot, w)$ is analytic for all $w \in \mathbb{C}^{n}$. By the same lemma and the formula

$$
G_{f}(z, w)=e^{-z \cdot w} \int_{\mathbb{C}^{n}} f(\bar{\zeta}) e^{z \cdot \zeta+w \cdot \bar{\zeta}} d \mu(\zeta), \quad z, w \in \mathbb{C}^{n},
$$

derived by the change of variables $\zeta \mapsto \bar{\zeta}$, we deduce that $G_{f}(z, \cdot)$ is analytic for each $z \in \mathbb{C}^{n}$. Thus $G_{f}$ is entire.

We introduce the notation

$$
F_{u}(\zeta):=f(\zeta) e^{-\left(\|\zeta\|^{2}+\|\zeta-u\|^{2}\right) / 2}, \quad u, \zeta \in \mathbb{C}^{n} .
$$

We claim that $F_{u} \in L^{1}(V) \cap L^{2}(V)$ for all $u \in \mathbb{C}^{n}$. This is an immediate consequence of the assumptions imposed upon $f$, since

$$
\left|F_{u}(\zeta)\right|=e^{-\|u\|^{2} / 2}\left|f(\zeta) e_{u}(\zeta)\right| e^{-\|\zeta\|^{2}}, \quad u, \zeta \in \mathbb{C}^{n} .
$$


Consider the change of variables $(u, v) \mapsto(z, w)$ given by

$$
z=\frac{1}{2}(u+i v) \quad \text { and } \quad w=\frac{1}{2}(\bar{u}+i \bar{v}) .
$$

Then

$$
e^{-\left(\|z\|^{2}+\|w\|^{2}\right) / 2} G_{f}(z, w)=e^{-(i / 2) \operatorname{Re} u \cdot \bar{v}} c_{n} \widehat{F}_{u}(v),
$$

where $\widehat{F}_{u}$ stands for the complex Fourier transform of the function $F_{u}$, and $c_{n}$ is a constant depending only on $n$. Observe that

$$
\begin{aligned}
\int_{\mathbb{C}^{n}} \int_{\mathbb{C}^{n}}\left|F_{u}(\zeta)\right|^{2} d V(\zeta) d V & (u) \\
& =\int_{\mathbb{C}^{n}}|f(\zeta)|^{2} e^{-\|\zeta\|^{2}} \int_{\mathbb{C}^{n}} e^{-\|\zeta-u\|^{2}} d V(u) d V(\zeta) \\
& =\pi^{2 n} \int_{\mathbb{C}^{n}}|f(\zeta)|^{2} d \mu(\zeta), \quad u \in \mathbb{C}^{n} .
\end{aligned}
$$

Applying (3), the change of variables (2), the Plancherel theorem and (4) we see that

$$
\begin{aligned}
\int_{\mathbb{C}^{n}} \int_{\mathbb{C}^{n}}\left|G_{f}(z, w)\right|^{2} d \mu(z) d \mu(w) & =\pi^{-2 n} c_{n}^{2} \int_{\mathbb{C}^{n}} \int_{\mathbb{C}^{n}}\left|\widehat{F}_{u}(v)\right|^{2} d V(z) d V(w) \\
& =d_{n} \int_{\mathbb{C}^{n}} \int_{\mathbb{C}^{n}}\left|\hat{F}_{u}(v)\right|^{2} d V(v) d V(u) \\
& =d_{n} \int_{\mathbb{C}^{n}} \int_{\mathbb{C}^{n}}\left|F_{u}(v)\right|^{2} d V(v) d V(u) \\
& =\pi^{2 n} d_{n} \int_{\mathbb{C}^{n}}|f(\zeta)|^{2} d \mu(\zeta),
\end{aligned}
$$

where $d_{n}$ is another constant depending only on $n$. Substituting $f \equiv 1$, which satisfies the assumptions of the theorem, we infer that

$$
G_{f}(z, w)=e^{-z \cdot w}\left\langle e_{\bar{w}}, e_{z}\right\rangle=1, \quad z, w \in \mathbb{C}^{n},
$$

thus $\pi^{2 n} d_{n}=1$.

Lemma 2 is a refined version of [9, Lemma 2.4], which can also serve as a prototype of the next lemma.

Lemma 3. Suppose that $f, \varphi: \mathbb{C}^{n} \rightarrow \mathcal{H}$ are Borel functions such that

$$
\|\varphi(\cdot)\| \in \bigcap_{a>0} X_{a} \quad \text { and } \quad\|f(z)\| \leq c e^{\|z\|^{2} / 2}, \quad z \in \mathbb{C}^{n},
$$

with some constant $c \geq 0$. Then the mapping $G$ given by

$$
G(z, w)=e^{-z \cdot w} \int_{\mathbb{C}^{n}}\langle f(\zeta), \varphi(\zeta)\rangle e^{z \cdot \bar{\zeta}+w \cdot \zeta} d \mu(\zeta)
$$


belongs to $\mathcal{B}_{2 n}$ if and only if $\langle f(\cdot), \varphi(\cdot)\rangle \in L^{2}(\mu)$. Moreover,

$$
\int_{\mathbb{C}^{n}}|\langle f(\zeta), \varphi(\zeta)\rangle|^{2} d \mu(\zeta)=\int_{\mathbb{C}^{n}} \int_{\mathbb{C}^{n}}|G(z, w)|^{2} d \mu(z) d \mu(w),
$$

with both integrals possibly infinite.

Proof. It suffices to check that the function $\langle f(\cdot), \varphi(\cdot)\rangle$ satisfies the assumptions of Lemma 2. Pick $a \in \mathbb{C}^{n}$ and fix any $D>\|a\|$. Then

$$
\begin{aligned}
\left|\langle f(\zeta), \varphi(\zeta)\rangle e_{a}(\zeta)\right| & \leq\|f(\zeta)\| \cdot\|\varphi(\zeta)\| e^{\|a\| \cdot\|\zeta\|} \\
& \leq M c e^{\|\zeta\|^{2}} e^{-(D-\|a\|)\|\zeta\|}, \quad \zeta \in \mathbb{C}^{n}
\end{aligned}
$$

where $M:=\sup _{\zeta \in \mathbb{C}^{n}}\|\varphi(\zeta)\| e^{D\|\zeta\|-\|\zeta\|^{2} / 2}<\infty$. This inequality implies that $\langle f(\cdot), \varphi(\cdot)\rangle e_{a}(\cdot) \in L^{1}(\mu)$. Moreover,

$$
\left|\langle f(\zeta), \varphi(\zeta)\rangle e_{a}(\zeta)\right|^{2} e^{-\|\zeta\|^{2}} \leq M^{2} c^{2} e^{\|\zeta\|^{2}} e^{-2(D-\|a\|)\|\zeta\|}, \quad \zeta \in \mathbb{C}^{n},
$$

so we infer that $\left|\langle f(\cdot), \varphi(\cdot)\rangle e_{a}(\cdot)\right| e^{-\|\cdot\|^{2} / 2} \in L^{2}(\mu)$.

Let us introduce the notation

$$
\Lambda_{\varphi} f(z):=\int_{\mathbb{C}^{n}}\langle f(\zeta), \varphi(\zeta)\rangle e^{z \cdot \bar{\zeta}} d \mu(\zeta), \quad z \in \mathbb{C}^{n}
$$

for all Borel functions $f, \varphi: \mathbb{C}^{n} \rightarrow \mathcal{H}$ for which the above integral is well defined for all $z \in \mathbb{C}^{n}$.

Lemma 4. Let $\varphi: \mathbb{C}^{n} \rightarrow \mathcal{H}$ be analytic such that $\|\varphi(\cdot)\| \in \bigcap_{a>0} X_{a}$. Then for any two fixed $z, w \in \mathbb{C}^{n}$ the following (well-defined) linear functionals are continuous ( $j$ being a multiindex):

(i) $\mathcal{B} \otimes \mathcal{H} \ni f \mapsto \Lambda_{\varphi}\left(e_{\bar{w}} f\right)(z) \in \mathbb{C}$,

(ii) $\mathcal{B} \otimes \mathcal{H} \ni f \mapsto \sum_{j \geq 0} \frac{w^{j}}{j !} \Lambda_{D^{j} \varphi} f(z) \in \mathbb{C}$.

Proof. (i) We prove simultaneously that the functional is well defined and continuous. Indeed, since $\|f(z)\| \leq\|f\|_{(\mathcal{H})} e^{\|z\|^{2} / 2}, z \in \mathbb{C}^{n}$, we have

$$
\int_{\mathbb{C}^{n}}\left|\langle f(\zeta), \varphi(\zeta)\rangle e^{w \cdot \zeta} e^{z \cdot \bar{\zeta}}\right| d \mu(\zeta) \leq\|f\|_{(\mathcal{H})} \int_{\mathbb{C}^{n}}\|\varphi(\zeta)\| \cdot\left|e^{w \cdot \zeta} e^{z \cdot \bar{\zeta}}\right| e^{-\|\zeta\|^{2} / 2} d V(\zeta) .
$$

The latter integral is convergent, since $\varphi$ is dominated by $e^{\|z\|^{2} / 2-C\|z\|}$ with any $C \geq 0$.

(ii) We will show that for any $C>0$ there exists $C^{\prime} \geq 0$ such that

$$
\int_{\mathbb{C}^{n}}\left|\left\langle f(\zeta), D^{j} \varphi(\zeta)\right\rangle e^{z \cdot \bar{\zeta}}\right| d \mu(\zeta) \leq C^{\prime}\|f\|_{(\mathcal{H})} \int_{\mathbb{C}^{n}}\left|\zeta^{j} e^{z \cdot \bar{\zeta}}\right| e^{-C\|\zeta\|} d V(\zeta)
$$

for all $j \in \mathbb{N}^{n}$. Note that this implies that $\Lambda_{D^{j} \varphi} f(z)$ is well defined for each $j \in \mathbb{N}^{n}$. Observe that for fixed $a \in \mathbb{C}^{n}$ and $\alpha>0$, the change of variables 
$x=\sqrt{\alpha} \zeta$ yields

$$
\begin{aligned}
\int_{\mathbb{C}^{n}}\left|e_{a}(\zeta)\right| e^{-\alpha\|\zeta\|^{2}} d V(\zeta) & =\frac{1}{\alpha^{n}} \int_{\mathbb{C}^{n}}\left|e_{a}\left(\frac{x}{\sqrt{\alpha}}\right)\right| e^{-\|x\|^{2}} d V(x) \\
& =\frac{1}{\alpha^{n}}\left\|e_{a /(2 \sqrt{\alpha})}\right\|^{2}=\frac{1}{\alpha^{n}} e^{\|a\|^{2} /(4 \alpha)} .
\end{aligned}
$$

In order to prove (5) we need to know that

$$
\left\langle f(\zeta), D^{j} \varphi(\zeta)\right\rangle=\int_{\mathbb{C}^{n}} \eta^{j}\langle f(\zeta), \varphi(\eta)\rangle e^{\bar{\zeta} \cdot \eta} d \mu(\eta), \quad \zeta \in \mathbb{C}^{n},
$$

which follows from Lemma 1. This implies that

$$
\int_{\mathbb{C}^{n}}\left|\left\langle f(\zeta), D^{j} \varphi(\zeta)\right\rangle e^{z \cdot \bar{\zeta}}\right| d \mu(\zeta) \leq \int_{\mathbb{C}^{n}} \int_{\mathbb{C}^{n}}|F(\zeta, \eta)| d \mu(\eta) d \mu(\zeta),
$$

where

$$
F(\zeta, \eta):=\eta^{j} e^{\zeta \cdot \bar{\eta}}\langle f(\zeta), \varphi(\eta)\rangle e^{\zeta \cdot \bar{z}}, \quad \zeta, \eta \in \mathbb{C}^{n} .
$$

Now, (6) yields

$$
\begin{aligned}
\int_{\mathbb{C}^{n}}|F(\zeta, \eta)| d \mu(\zeta) & \leq\|f\|_{(\mathcal{H})}\left|\eta^{j}\right| \cdot\|\varphi(\eta)\| \int_{\mathbb{C}^{n}}\left|e^{\zeta \cdot(\bar{\eta}+\bar{z})}\right| e^{-\|\zeta\|^{2} / 2} d V(\zeta) \\
& =2^{n}\|f\|_{(\mathcal{H})}\left|\eta^{j}\right| \cdot\|\varphi(\eta)\| e^{\|\eta+z\|^{2} / 2} \\
& \leq C^{\prime}\|f\|_{(\mathcal{H})}\left|\eta^{j}\right| e^{-C\|\eta\|+\|\eta\|^{2} / 2-\|z\|^{2} / 2+\|\eta+z\|^{2} / 2}, \quad \eta \in \mathbb{C}^{n},
\end{aligned}
$$

with arbitrary $C>0$ and another constant $C^{\prime} \geq 0$ chosen so that

$$
e^{-\|z\|^{2} / 2} 2^{-n} C^{\prime} \geq\|\varphi(\eta)\| e^{C\|\eta\|-\|\eta\|^{2} / 2}
$$

for all $\eta \in \mathbb{C}^{n}$. Note that $C^{\prime}$ does not depend on $j$. Hence we infer that

$$
\int_{\mathbb{C}^{n}} \int_{\mathbb{C}^{n}}|F(\zeta, \eta)| d \mu(\zeta) d \mu(\eta) \leq C^{\prime}\|f\|_{(\mathcal{H})} \int_{\mathbb{C}^{n}}\left|\eta^{j} e^{\eta \cdot \bar{z}}\right| e^{-C\|\eta\|} d V(\eta)
$$

which combined with (7) gives (5).

Once (5) is established we can prove that the functional defined in (ii) is continuous. Fix $C>\|z\|+\|w\|$ and choose $C^{\prime} \geq 0$ so that (5) holds. Then

$$
\begin{aligned}
\sum_{j \geq 0} \frac{\left|w^{j}\right|}{j !}\left|\Lambda_{D^{j} \varphi} f(z)\right| & \leq C^{\prime}\|f\|_{(\mathcal{H})} \sum_{j \geq 0} \frac{\left|w^{j}\right|}{j !} \int_{\mathbb{C}^{n}}\left|\zeta^{j} e^{\zeta \cdot \bar{z}}\right| e^{-C\|\zeta\|} d V(\zeta) \\
& =C^{\prime}\|f\|_{(\mathcal{H})} \int_{\mathbb{C}^{n}}\left(\sum_{j \geq 0} \frac{\left|w^{j} \zeta^{j}\right|}{j !}\right)\left|e^{\zeta \cdot \bar{z}}\right| e^{-C\|\zeta\|} d V(\zeta) \\
& =C^{\prime}\|f\|_{(\mathcal{H})} \int_{\mathbb{C}^{n}} e^{\left|w_{1} \zeta_{1}\right|+\ldots+\left|w_{n} \zeta_{n}\right|}\left|e^{\zeta \cdot \bar{z}}\right| e^{-C\|\zeta\|} d V(\zeta) .
\end{aligned}
$$


The latter integral is convergent since

$$
\sum_{k=1}^{n}\left|w_{k} \zeta_{k}\right|+\operatorname{Re} \zeta \cdot \bar{z}-C\|\zeta\| \leq-(C-\|z\|-\|w\|)\|\zeta\|, \quad w, z, \zeta \in \mathbb{C}^{n} .
$$

In the following proof we put $\varphi_{h}(z):=\langle\varphi(z), h\rangle$ for $\varphi: \mathbb{C}^{n} \rightarrow \mathcal{H}$ and $h \in \mathcal{H}$.

Lemma 5. Let $\varphi: \mathbb{C}^{n} \rightarrow \mathcal{H}$ be an analytic function such that $\|\varphi(\cdot)\| \in$ $\bigcap_{a>0} X_{a}$. Then

$$
e^{-z \cdot w} \Lambda_{\varphi}\left(e_{\bar{w}} f\right)(z)=\sum_{j \geq 0} \frac{w^{j}}{j !} \Lambda_{D^{j} \varphi} f(z)
$$

for all $f \in \mathcal{B} \otimes \mathcal{H}$ and $z, w \in \mathbb{C}^{n}$.

Proof. Choose $z, w \in \mathbb{C}^{n}$. By Lemma 4 the formula (8) can be regarded as equality of two continuous linear functionals defined for $f \in \mathcal{B} \otimes \mathcal{H}$. Thus it suffices to check (8) only for $f:=e_{a} \otimes h, a \in \mathbb{C}^{n}, h \in \mathcal{H}$. Since $e_{z} \varphi_{h} \in \mathcal{B}$, by the reproducing property of $\mathcal{B}$ we see that

$$
e^{-z \cdot w} \Lambda_{\varphi}\left(e_{\bar{w}} f\right)(z)=e^{-z \cdot w}\left\langle e_{\bar{w}+a}, e_{z} \varphi_{h}\right\rangle=\overline{\varphi_{h}(\bar{w}+a)} e^{z \cdot \bar{a}} .
$$

On the other hand,

$$
\Lambda_{D^{j} \varphi} f(z)=\int_{\mathbb{C}^{n}} e_{a}(\zeta) \overline{\psi_{j}(\zeta)} d \mu(\zeta), \quad j \in \mathbb{N}^{n},
$$

where $\psi_{j}(\zeta):=e^{\zeta \cdot \bar{z}} D^{j} \varphi_{h}(\zeta), \zeta \in \mathbb{C}^{n}, j \in \mathbb{N}^{n}$. But $\varphi_{h} \in \mathcal{B}$, so by Lemma 1 ,

$$
\Lambda_{D^{j} \varphi} f(z)=\overline{\psi_{j}(a)}=\overline{D^{j} \varphi_{h}(a)} e^{z \cdot \bar{a}} .
$$

To complete the proof of (8) we have to verify the equality

$$
\overline{\varphi_{h}(\bar{w}+a)} e^{z \cdot \bar{a}}=\sum_{j \geq 0} \frac{w^{j}}{j !} \overline{D^{j} \varphi_{h}(a)} e^{z \cdot \bar{a}}
$$

which follows from the Taylor expansion of $\varphi_{h}$ at $a$.

3. The isometry theorems. In this section we give several formulations of the generalized Newman-Shapiro Isometry Theorem, each of which can be regarded as an extension of theorems proved in [22] to the case of vector-valued functions. Let $\varphi^{*}$ be defined by $\varphi^{*}(z):=(\varphi(z))^{*}$. We "extend" the symbol $\Lambda$ by putting

$$
\widetilde{\Lambda}_{\varphi} f(z):=\int_{\mathbb{C}^{n}} \varphi^{*}(\zeta) f(\zeta) e^{z \cdot \bar{\zeta}} d \mu(\zeta), \quad z \in \mathbb{C}^{n},
$$

for all Borel functions $f: \mathbb{C}^{n} \rightarrow \mathcal{H}$ and $\varphi: \mathbb{C}^{n} \rightarrow B(\mathcal{K}, \mathcal{H})$ for which the above integral is well defined for each $z \in \mathbb{C}^{n}$. Applying Lemma 4 one can show that if $\varphi$ is analytic and $\|\varphi(\cdot)\| \in \bigcap_{a>0} X_{a}$, then for every $f \in \mathcal{B} \otimes \mathcal{H}$, 
$j \in \mathbb{N}^{n}$ and $z \in \mathbb{C}^{n}$ the integral which defines $\widetilde{\Lambda}_{D^{j} \varphi} f(z)$ is convergent. Indeed, it suffices to check that for every fixed $h \in \mathcal{H}$,

$$
\left\langle\widetilde{\Lambda}_{D^{j} \varphi} f(z), h\right\rangle=\Lambda_{D^{j} \varphi(\cdot) h} f(z), \quad z \in \mathbb{C}^{n}, j \in \mathbb{N}^{n},
$$

and $\|\varphi(\cdot) h\| \in \bigcap_{a>0} X_{a}$.

Theorem 6. Suppose that $\varphi: \mathbb{C}^{n} \rightarrow B(\mathcal{K}, \mathcal{H})$ is an analytic function such that $\|\varphi(\cdot)\| \in \bigcap_{a>0} X_{a}$. Then

$$
\left\|\varphi^{*} f\right\|_{(\mathcal{K})}^{2}=\sum_{j \geq 0} \frac{1}{j !}\left\|\widetilde{\Lambda}_{D^{j} \varphi} f\right\|_{(\mathcal{K})}^{2}
$$

for $f \in \mathcal{B} \otimes \mathcal{H}$ (with the convention that $\|H\|_{(\mathcal{K})}=\infty$ whenever $H \notin L^{2}(\mu)$ $\otimes \mathcal{K})$.

Proof. We first focus on a special case of the theorem. For $f, \psi: \mathbb{C}^{n} \rightarrow \mathcal{H}$ analytic and such that $f \in \mathcal{B} \otimes \mathcal{H}$ and $\|\psi(\cdot)\| \in \bigcap_{a>0} X_{a}$, we show that

$$
\|\langle f(\cdot), \psi(\cdot)\rangle\|^{2}=\sum_{j \geq 0} \frac{1}{j !}\left\|\Lambda_{D^{j} \psi} f\right\|^{2}
$$

(with the convention that $\|F\|=\infty$ whenever $F \notin L^{2}(\mu)$ ).

Let

$$
G(z, w):=e^{-z \cdot w} \int_{\mathbb{C}^{n}}\langle f(\zeta), \psi(\zeta)\rangle e^{z \cdot \bar{\zeta}+w \cdot \zeta} d \mu(\zeta), \quad z, w \in \mathbb{C}^{n},
$$

that is, $G(z, w)=e^{-z \cdot w} \Lambda_{\psi}\left(e_{\bar{w}} f\right)(z)$. By Lemma 5 ,

$$
G(z, w)=\sum_{j \geq 0} \frac{w^{j}}{j !} \Lambda_{D^{j} \psi} f(z), \quad z, w \in \mathbb{C}^{n} .
$$

For fixed $z \in \mathbb{C}^{n}$, both sides of the above equality represent scalar functions, analytic in $w$. Hence

$$
\int_{\mathbb{C}^{n}}|G(z, w)|^{2} d \mu(w)=\sum_{j \geq 0} \frac{1}{j !}\left|\Lambda_{D^{j} \psi} f(z)\right|^{2} .
$$

Integrating with respect to $d \mu(z)$ and applying Lemma 3 we obtain the desired equality.

We now turn to the general case. Let $\left\{\varepsilon_{k}\right\}_{k=0}^{\infty}$ be an orthonormal basis for the Hilbert space $\mathcal{K}$. Then

$$
\left\|\varphi^{*} f\right\|_{(\mathcal{K})}^{2}=\sum_{k=0}^{\infty}\left\|\left(\varphi^{*} f\right)_{\varepsilon_{k}}\right\|^{2}
$$

where $\left(\varphi^{*} f\right)_{\varepsilon_{k}}(z):=\left\langle\varphi^{*}(z) f(z), \varepsilon_{k}\right\rangle$. Similarly,

$$
\left\|\widetilde{\Lambda}_{D^{j} \varphi} f\right\|_{(\mathcal{K})}^{2}=\sum_{k=0}^{\infty}\left\|\left(\widetilde{\Lambda}_{D^{j} \varphi} f\right)_{\varepsilon_{k}}\right\|^{2}, \quad j \in \mathbb{N}^{n}
$$


(with the convention that $\|F\|=\infty$ whenever $F \notin L^{2}(\mu)$ ). So it suffices to show that

$$
\left\|\left(\varphi^{*} f\right)_{\varepsilon_{k}}\right\|^{2}=\sum_{j \geq 0} \frac{1}{j !}\left\|\left(\widetilde{\Lambda}_{D^{j} \varphi} f\right)_{\varepsilon_{k}}\right\|^{2}
$$

for all $k \in \mathbb{N}^{n}$; but this follows from the special case of the theorem.

The above theorem can easily be translated into an "operator version". In order to do it, we need to define an extended Toeplitz operator $\Pi_{\varphi}$ with $\varphi: \mathbb{C}^{n} \rightarrow B(\mathcal{K}, \mathcal{H})$ being a Borel function (in a weak sense). The domain of $\Pi_{\varphi}$ consists of all $f \in \mathcal{B} \otimes \mathcal{H}$ such that the integral

$$
\Pi_{\varphi^{*}} f(z):=\int_{\mathbb{C}^{n}} \varphi^{*}(\zeta) f(\zeta) e^{z \cdot \zeta} d \mu(\zeta), \quad z \in \mathbb{C}^{n}
$$

exists (i.e. it is weakly convergent, cf. [9]) for all $z \in \mathbb{C}^{n}$ and the function of $z$ defined by it lies in $\mathcal{B} \otimes \mathcal{K}$. If $f \in \mathcal{D}\left(\Pi_{\varphi}\right)$, then the above formula defines $\Pi_{\varphi} f$.

Theorem 7. Assume that $\varphi: \mathbb{C}^{n} \rightarrow B(\mathcal{K}, \mathcal{H})$ is an analytic function such that $\|\varphi(\cdot)\| \in \bigcap_{a>0} X_{a}$. Then

$$
\left\|\varphi^{*} f\right\|_{(\mathcal{K})}^{2}=\sum_{j \geq 0} \frac{1}{j !}\left\|\Pi_{\left(D^{j} \varphi\right)^{*}} f\right\|_{(\mathcal{K})}^{2}
$$

for $f \in \mathcal{B} \otimes \mathcal{H}$ (with the convention that $\|H\|_{(\mathcal{K})}=\infty$ whenever $H \notin L^{2}(\mu)$ $\otimes \mathcal{K}$ or $\left.H \notin \mathcal{D}\left(\Pi_{\left(D^{j} \varphi\right)^{*}}\right)\right)$.

Proof. This is an immediate consequence of Theorem 6.

A special case of Theorem 7 is when $\varphi$ is a polynomial, which always satisfies the assumption of this theorem. Let $p \in \mathcal{P} \otimes B(\mathcal{K}, \mathcal{H})$. We know that $\Pi_{p^{*}}=p^{\#}(D)$, where $p^{\#}(D)$ is understood as an unbounded operator acting between $\mathcal{B} \otimes \mathcal{H}$ and $\mathcal{B} \otimes \mathcal{K}$ with domain $\mathcal{D}\left(p^{\#}(D)\right):=\{f \in \mathcal{B} \otimes \mathcal{H}$ : $\left.p^{\#}(D) f \in \mathcal{B} \otimes \mathcal{K}\right\}$ (cf. [9, Proposition 6.1] as well as Corollary 10 below). The isometry theorem can then be rephrased as

$$
\left\|p^{*} f\right\|_{(\mathcal{K})}^{2}=\sum_{j \geq 0} \frac{1}{j !}\left\|\left(D^{j} p^{\#}\right)(D) f\right\|_{(\mathcal{K})}^{2}
$$

for $f \in \mathcal{B} \otimes \mathcal{H}$ (with the usual convention). This equality was given in [22] in the scalar case, and its full version appeared in [9].

One more version of the isometry theorem can be derived from Lemma 3.

TheOREM 8. Let $\varphi: \mathbb{C}^{n} \rightarrow B(\mathcal{K}, \mathcal{H})$ be a Borel function such that $\|\varphi(\cdot)\| \in \bigcap_{a>0} X_{a}$ and $f \in \mathcal{B} \otimes \mathcal{H}$. Then the mapping $G$ given by

$$
G(z, w)=e^{-z \cdot w} \int_{\mathbb{C}^{n}} \varphi^{*}(\zeta) f(\zeta) e^{z \cdot \bar{\zeta}+w \cdot \zeta} d \mu(\zeta)
$$


belongs to $\mathcal{B}_{2 n} \otimes \mathcal{K}$ if and only if $\left\|\varphi^{*}(\cdot) f(\cdot)\right\| \in L^{2}(\mu)$. Moreover,

$$
\int_{\mathbb{C}^{n}}\left\|\varphi^{*}(\zeta) f(\zeta)\right\|^{2} d \mu(\zeta)=\int_{\mathbb{C}^{n}} \int_{\mathbb{C}^{n}}\|G(z, w)\|^{2} d \mu(z) d \mu(w),
$$

with both integrals possibly infinite.

Proof. Fix an orthonormal basis $\left\{\varepsilon_{k}\right\}_{k=0}^{\infty}$ for $\mathcal{K}$. Observe that $\psi_{k}(z):=$ $\varphi(z) \varepsilon_{k}$ satisfies the conditions imposed on $\varphi$ in Lemma 3. So we can write

$$
\int_{\mathbb{C}^{n}}\left|\left\langle f(\zeta), \psi_{k}(\zeta)\right\rangle\right|^{2} d \mu(\zeta)=\int_{\mathbb{C}^{n}} \int_{\mathbb{C}^{n}}\left|\left\langle G(z, w), \varepsilon_{k}\right\rangle\right|^{2} d \mu(z) d \mu(w)
$$

for all $k \geq 0$. Summation over all $k$ 's completes the proof.

4. Adjointness. Let $\varphi: \mathbb{C}^{n} \rightarrow B(\mathcal{H}, \mathcal{K})$ be an analytic function. An analytic Toeplitz operator with symbol $\varphi$ is defined by $\mathcal{D}\left(T_{\varphi}\right)=\{f \in \mathcal{B} \otimes \mathcal{H}$ : $\varphi f \in \mathcal{B} \otimes \mathcal{K}\}$ and $T_{\varphi} f=\varphi f$ for $f \in \mathcal{D}\left(T_{\varphi}\right)$, where $(\varphi f)(z)=\varphi(z) f(z)$, $z \in \mathbb{C}^{n}$. One of the basic questions concerning Toeplitz operators, which remains unanswered, is whether $T_{\varphi}^{*}=\Pi_{\varphi^{*}}$ for all analytic symbols $\varphi$, which is referred to as the adjointness hypothesis. Some partial positive results were proved, e.g. for exponential polynomials in the scalar case $(\mathcal{H}=\mathcal{K}=\mathbb{C})$ [22] or matrix-valued polynomials in one complex variable [10]. For a more detailed exposition of this subject see [10].

Our goal is to prove the adjointness hypothesis for special operatorvalued exponential polynomials, i.e. functions of the type

$$
\varphi(z)=\sum_{|k| \leq N} A_{k} p_{k}(z) e_{a_{k}}(z), \quad z \in \mathbb{C}^{n},
$$

where $A_{k} \in B(\mathcal{K}, \mathcal{H}), p_{k} \in \mathcal{P}$ and $a_{k} \in \mathbb{C}^{n}$ for $|k| \leq N$.

We will consider analytic functions $\varphi: \mathbb{C}^{n} \rightarrow B(\mathcal{K}, \mathcal{H})$ which have the following summability property (SP): given $f \in \mathcal{D}\left(T_{\varphi}\right)$ and $g \in \mathcal{D}\left(\Pi_{\varphi^{*}}\right)$, the function

$$
\mathbb{C}^{n} \times \mathbb{C}^{n} \ni(z, w) \mapsto\langle g(w), \varphi(w) f(z)\rangle e^{z \cdot \bar{w}} \in \mathbb{C}
$$

is summable with respect to $\mu \otimes \mu$. Observe that $T_{\varphi}^{*}=\Pi_{\varphi^{*}}$ whenever $\varphi$ satisfies (SP). Indeed, for $f \in \mathcal{D}\left(T_{\varphi}\right)$ and $g \in \mathcal{D}\left(\Pi_{\varphi^{*}}\right)$ we can write

$$
\begin{aligned}
\left\langle T_{\varphi} f, g\right\rangle_{(\mathcal{H})} & =\int_{\mathbb{C}^{n}}\left\langle f(z), \varphi(z)^{*} g(z)\right\rangle d \mu(z) \\
& =\int_{\mathbb{C}^{n}} \int_{\mathbb{C}^{n}}\left\langle f(w), \varphi(z)^{*} g(z)\right\rangle e^{z \cdot \bar{w}} d \mu(w) d \mu(z) \\
& \stackrel{F}{=} \int_{\mathbb{C}^{n}} \int_{\mathbb{C}^{n}}\left\langle f(w), \varphi(z)^{*} g(z)\right\rangle e^{z \cdot \bar{w}} d \mu(z) d \mu(w) \\
& =\left\langle f, \Pi_{\varphi^{*}} g\right\rangle_{(\mathcal{H})},
\end{aligned}
$$


where " $\stackrel{F}{=}$ " denotes an application of the Fubini theorem, which is possible by (SP).

It now remains to prove (SP) for the class of exponential polynomials. Recall that a family $\left\{A_{\imath}\right\}_{\imath \in I}$ of bounded operators on $\mathcal{H}$ is called jointly subnormal if there exists a family $\left\{B_{\imath}\right\}_{\imath \in I}$ of normal commuting bounded operators on a larger Hilbert space $\mathcal{K}$ containing $\mathcal{H}$ as a closed subspace such that $A_{\imath}=\left.B_{\imath}\right|_{\mathcal{H}}$ for all $\imath \in I$. Note that by the minimality condition on $\mathcal{K}$, if $I$ is finite and $\mathcal{H}$ is separable, then $\mathcal{K}$ can be chosen to be separable, too.

Proposition 9. Suppose that $\varphi(z)=\sum_{|k| \leq N} A_{k} p_{k}(z) e_{a_{k}}(z), z \in \mathbb{C}^{n}$, $p_{k} \in \mathcal{P}$ and $A_{k} \in B(\mathcal{H})$ are jointly subnormal. Then $\varphi$ satisfies (SP).

Proof. Let $f$ and $g$ be as in (9). The change of variables $w=z+a$ yields

$$
\int_{\mathbb{C}^{n}}\left|\langle g(w), \varphi(w) f(z)\rangle e^{z \cdot \bar{w}}\right| d \mu(w)=\int_{\mathbb{C}^{n}}\left|\langle g(z+a), \varphi(z+a) f(z)\rangle e^{-z \cdot \bar{a}}\right| d \mu(a) .
$$

Thus

$$
\begin{aligned}
J & :=\int_{\mathbb{C}^{n}} \int_{\mathbb{C}^{n}}\left|\langle g(w), \varphi(w) f(z)\rangle e^{z \cdot \bar{w}}\right| d \mu(w) d \mu(z) \\
& \leq \int_{\mathbb{C}^{n}}\left\|g(\cdot+a) e_{-a}(\cdot)\right\|_{(\mathcal{H})}\|\varphi(\cdot+a) f(\cdot)\|_{(\mathcal{H})} d \mu(a) .
\end{aligned}
$$

But $\left\|g(\cdot+a) e_{-a}(\cdot)\right\|_{(\mathcal{H})}=e^{\|a\|^{2} / 2}\|g\|_{(\mathcal{H})}$, which implies that

$$
J \leq\|g\|_{(\mathcal{H})} \int_{\mathbb{C}^{n}}\|\varphi(\cdot+a) f(\cdot)\|_{(\mathcal{H})} e^{\|a\|^{2} / 2} d \mu(a) .
$$

Let $\left\{B_{k}\right\}_{|k|<N} \subseteq B(\mathcal{K})$ be a family of normal commuting operators such that $A_{k}=\left.B_{k}\right|_{\mathcal{H}}$. Put $\psi(z):=\sum_{|k| \leq N} B_{k} p_{k}(z) e_{a_{k}}(z), z \in \mathbb{C}^{n}$. Observe that every $\psi(z)$ is a normal operator, $z \in \mathbb{C}^{n}$. Moreover, $f \in \mathcal{D}\left(T_{\psi}\right)$ and $\|\psi(\cdot+a) f(\cdot)\|_{(\mathcal{K})}=\|\varphi(\cdot+a) f(\cdot)\|_{(\mathcal{H})}$, since $\varphi(z) h=\psi(z) h$ for $z \in \mathbb{C}^{n}$ and $h \in \mathcal{H}$. Thus, in order to prove that $J<\infty$ we have to show that

$$
\int_{\mathbb{C}^{n}}\left\|\psi^{*}(\cdot+a) f(\cdot)\right\|_{(\mathcal{K})} e^{\|a\|^{2} / 2} d \mu(a)<\infty .
$$

Let $G(z, w):=e^{z \cdot w} \int_{\mathbb{C}^{n}} \psi(\zeta)^{*} f(\zeta) e^{z \cdot \bar{\zeta}+w \cdot \zeta} d \mu(\zeta)$. According to Theorem 8 we know that $\int_{\mathbb{C}^{n}} \int_{\mathbb{C}^{n}}\|G(z, w)\|^{2} d \mu(z) d \mu(w)=\left\|\psi^{*} f\right\|_{(\mathcal{K})}^{2}<\infty$. Fix $h \in \mathcal{K}$ and compute

$$
\langle G(z, w), h\rangle=e^{-z \cdot w}\left\langle f e_{\bar{w}}, \psi h e_{z}\right\rangle_{(\mathcal{K})}=e^{-z \cdot w}\left\langle f, P \otimes I\left(\bar{e}_{\bar{w}} \psi h e_{z}\right)\right\rangle_{(\mathcal{K})} .
$$

By the reproducing property of $\mathcal{B} \otimes \mathcal{K}$,

$$
P \otimes I\left(\bar{e}_{\bar{w}} \psi h e_{z}\right)(\zeta)=\int_{\mathbb{C}^{n}} \psi(t) h e^{t \cdot \bar{z}} e^{(\bar{w}+\zeta) \cdot \bar{t}} d \mu(t)=\psi(\bar{w}+\zeta) h e^{(\bar{w}+\zeta) \cdot \bar{z}}
$$


for $\zeta \in \mathbb{C}^{n}$, which implies that

$$
\begin{aligned}
G(z, w) & =\int_{\mathbb{C}^{n}} \psi(\bar{w}+\zeta)^{*} f(\zeta) e^{z \cdot \bar{\zeta}} d \mu(\zeta) \\
& =\sum_{|k| \leq N} \int_{\mathbb{C}^{n}} \overline{p_{k}(\bar{w}+\zeta) e^{(\bar{w}+\zeta) \cdot \bar{a}_{k}}} B_{k}^{*} f(\zeta) e^{z \cdot \bar{\zeta}} d \mu(\zeta) \\
& =\sum_{|k| \leq N} \sum_{l \geq 0} \int_{\mathbb{C}^{n}} e^{a_{k} \cdot w} \frac{\left(D^{l} p_{k}^{\#}\right)(w)}{l !} \bar{\zeta}^{l} B_{k}^{*} f(\zeta) e^{\left(z+a_{k}\right) \cdot \bar{\zeta}} d \mu(\zeta) \\
& =\sum_{|k| \leq N} \sum_{l \geq 0} e^{a_{k} \cdot w} \frac{\left(D^{l} p_{k}^{\#}\right)(w)}{l !} B_{k}^{*}\left(D^{l} f\right)\left(z+a_{k}\right)
\end{aligned}
$$

for all $z, w \in \mathbb{C}^{n}$ (apply Lemma 1 ).

For fixed $k$ choose a maximal linearly independent set of functions contained in $\left\{D^{l} p_{s}^{\#}: l \geq 0, a_{s}=a_{k}\right\}$; denote it by $\left\{p_{k j}: j=1, \ldots, N_{k}\right\}$. Note that we can write

$$
G(z, w)=\sum_{|k| \leq N} \sum_{j=1}^{N_{k}} e^{a_{k} \cdot w} p_{k j}(w) F_{k j}(z), \quad z, w \in \mathbb{C}^{n},
$$

where every $F_{k j}(\cdot)$ is a linear combination of properly chosen $B_{s}^{*}\left(D^{l} f\right)\left(\cdot+a_{s}\right)$. We intend to show that each $F_{k j}$ belongs to $\mathcal{B} \otimes \mathcal{K}$. We first prove that $\bigcup_{|k| \leq N}\left\{e_{a_{k}} p_{k j}: j=1, \ldots, N_{k}\right\}$ is a set of linearly independent vectors. Suppose that

$$
\sum_{|k| \leq N} \sum_{j=1}^{N_{k}} \lambda_{k j} e_{a_{k}} p_{k j}=0
$$

with some $\lambda_{k j} \in \mathbb{C}$. Hence $\sum_{|k| \leq N} e_{a_{k}} q_{k}=0$, where $q_{k}:=\sum_{j=1}^{N_{k}} \lambda_{k j} p_{k j}$. This implies that $q_{k}=0$ for all $k$, which holds true in all generality with arbitrary polynomials $q_{k}$ (the details are left to the reader). By definition of $p_{k j}$ we see that all $\lambda_{k j}$ must vanish.

Fix $k$ and $j$. Then one can construct a function $h_{k j} \in \mathcal{B}$ such that $\left\|h_{k j}\right\|=1, h_{k j} \perp e_{a_{s}} p_{s t}$ if $(k, j) \neq(s, t)$, and $\left\langle h_{k j}, e_{a_{k}} p_{k j}\right\rangle>0$. Let $\varepsilon \in \mathcal{K}$ be an arbitrary vector with norm less than one. We infer that

$$
\begin{aligned}
\int_{\mathbb{C}^{n}}\|G(z, w)\|^{2} d \mu(w) & \geq\left|\left\langle G(z, \cdot), h_{k j}(\cdot) \varepsilon\right\rangle(\mathcal{K})\right|^{2} \\
& =\left|\int_{\mathbb{C}^{n}} \sum_{|s| \leq N} \sum_{t=1}^{N_{s}} e_{a_{s}}(w) p_{s t}(w) \overline{h_{k j}(w)}\left\langle F_{k j}(z), \varepsilon\right\rangle d \mu(w)\right|^{2} \\
& \geq\left\langle e_{a_{k}} p_{k j}, h_{k j}\right\rangle^{2}\left|\left\langle F_{k j}(z), \varepsilon\right\rangle\right|^{2},
\end{aligned}
$$

so it follows that $F_{k j} \in \mathcal{B} \otimes \mathcal{K}$. 
We now turn to the estimation of $\left\|\psi(\cdot+a)^{*} f(\cdot)\right\|_{(\mathcal{K})}$. Put $\widetilde{G}(z, w)=$ $e^{-z \cdot w} \int_{\mathbb{C}^{n}} \psi(\zeta+a)^{*} f(\zeta) e^{z \cdot \bar{\zeta}+w \cdot \zeta} d \mu(\zeta)$. Repeating the argument for $G$, we can write

$$
\widetilde{G}(z, w)=\sum_{|k| \leq N} \sum_{j=1}^{N_{k}} e^{a_{k} \cdot(w+\bar{a})} p_{k j}(w+\bar{a}) F_{k j}(z), \quad z, w \in \mathbb{C}^{n},
$$

with $p_{k j}$ and $F_{k j}$ as in (11). This implies that the growth of $\left\|\psi(\cdot+a)^{*} f(\cdot)\right\|_{(\mathcal{K})}$ is at most exponential in $a$, so the integral in (10) is finite.

Note that the above proof gives a slight strengthening of (SP), because we have used only the fact that $g \in \mathcal{B} \otimes \mathcal{H}$. Presumably, finding a method of recovering some information on $g$ which satisfies $g \in \mathcal{D}\left(\Pi_{\varphi^{*}}\right)$ would lead to progress in proving (or disproving) the adjointness hypothesis in a wider class of symbols $\varphi$.

We conclude the paper with a nice description of $T_{\varphi}^{*}$ for exponential polynomials. Let $\left(E_{a} f\right)(z)=f(z+a)$ for $f: \mathbb{C}^{n} \rightarrow \mathcal{H}$ and $a \in \mathbb{C}^{n}$.

Corollary 10. Let $\varphi$ be as in Proposition 9. Then

$$
T_{\varphi}^{*} f=\sum_{|k| \leq N} E_{a_{k}} p_{k}^{\#}(D) A_{k}^{*} f, \quad f \in \mathcal{D}\left(T_{\varphi}^{*}\right),
$$

and $\mathcal{D}\left(T_{\varphi}^{*}\right)$ coincides with the space of those $f \in \mathcal{B} \otimes \mathcal{H}$ for which the righthand side of (12) lies in $\mathcal{B} \otimes \mathcal{H}$.

Proof. Let $f \in \mathcal{D}\left(\Pi_{\varphi^{*}}\right)$ and $h \in \mathcal{H}$. Applying Lemma 1 we get

$$
\begin{aligned}
\left\langle\Pi_{\varphi^{*}} f(z), h\right\rangle & =\int_{\mathbb{C}^{n}}\langle f(\zeta), \varphi(\zeta) h\rangle e^{z \cdot \bar{\zeta}} d \mu(\zeta) \\
& =\sum_{|k| \leq N} \int_{\mathbb{C}^{n}} \overline{p_{k}(\zeta)}\left\langle f(\zeta), A_{k} h\right\rangle e^{\left(z+a_{k}\right) \cdot \bar{\zeta}} d \mu(\zeta) \\
& =\left\langle\sum_{|k| \leq N} E_{a_{k}} p_{k}^{\#}(D) A_{k}^{*} f(z), h\right\rangle
\end{aligned}
$$

for $z \in \mathbb{C}^{n}$. By Proposition 9 the result follows.

\section{References}

[1] V. Bargmann, On a Hilbert space of analytic functions and an associated integral transform, Comm. Pure Appl. Math. 14 (1961), 187-214.

[2] F. A. Berezin, Vick and anti-Vick symbols of operators, Mat. Sb. 86 (1971), 578-610 (in Russian).

[3] -, Covariant and contravariant symbols of operators, Math. USSR-Izv. 6 (1972), 1117-1151.

[4] C. A. Berger and L. A. Coburn, Toeplitz operators and quantum mechanics, J. Funct. Anal. 68 (1986), 273-299. 
[5] C. A. Berger and L. A. Coburn, Toeplitz operators on the Segal-Bargmann space, Trans. Amer. Math. Soc. 301 (1987), 813-829.

[6] —, - Heat flow and Berezin-Toeplitz estimates, Amer. J. Math. 116 (1994), 563590.

[7] S. B. Chae, Holomorphy and Calculus in Normed Spaces, Dekker, New York and Basel, 1985.

[8] D. Cichon, Notes on unbounded Toeplitz operators in Segal-Bargmann spaces, Ann. Polon. Math. 3 (1996), 227-235.

[9] —, Generalization of the Newman-Shapiro Isometry Theorem and Toeplitz operators, Integral Equations Operator Theory 34 (1999), 414-438.

[10] D. Cichoń and H. S. Shapiro, Toeplitz operators in Segal-Bargmann spaces of vectorvalued functions, submitted.

[11] D. Cichoń and J. Stochel, On Toeplitz operators in Segal-Bargmann spaces, Univ. Iagell. Acta Math. 34 (1997), 35-44.

[12] E. Fischer, Über die Differentiationsprozesse der Algebra, J. für Math. 148 (1917), $1-78$.

[13] V. Fock, Zur Quanten Elektrodynamik, Physikalische Zeitschrift der Sowjetunion 6 (1934), 425-469.

[14] V. Guillemin, Toeplitz operators in $n$ dimensions, Integral Equations Operator Theory 7 (1984), 145-205.

[15] J. Janas, Toeplitz and Hankel operators on Bargmann spaces, Glasgow Math. J. 30 (1988), 315-323.

[16] - Unbounded Toeplitz operators in the Bargmann-Segal space, Studia Math. 99 (1991), 87-99.

[17] -, An operator version of the Newman-Shapiro Isometry Theorem, Integral Equations Operator Theory 26 (1996), 188-201.

[18] —, The Newman-Shapiro isometry theorem-operator version for systems, Univ. Iagell. Acta Math. 34 (1997), 57-61.

[19] J. Janas and J. Stochel, Unbounded Toeplitz operators in the Segal-Bargmann space. II, J. Funct. Anal. 126 (1994), 418-447.

[20] - - - Unbounded Toeplitz operators in the Segal-Bargmann space, IV, in preparation.

[21] D. J. Newman and H. S. Shapiro, Certain Hilbert spaces of entire functions, Bull. Amer. Math. Soc. 72 (1966), 971-977.

[22] - - - Fischer spaces of entire functions, in: Entire Functions and Related Parts of Analysis, J. Korevaar (ed.), Proc. Sympos. Pure Math. 11, Amer. Math. Soc., Providence, RI, 1968, 360-369.

[23] I. E. Segal, Lectures at the Summer Seminar on Applied Mathematics, Boulder, CO, 1960.

Institute of Mathematics

Jagiellonian University

Reymonta 4

30-059 Kraków, Poland

E-mail: cichon@im.uj.edu.pl 